The Astrophysical Journal SupPlement Series, 90:857-861, 1994 February

(C) 1994. The American Astronomical Society. All rights reserved. Printed in U.S.A.

\title{
OBSERVATIONAL FEATURES OF COSMIC GAMMA-RAY BURSTS: EVIDENCE FOR GALACTIC VERSUS EXTRAGALACTIC ORIGIN
}

\author{
KeVIN HuRLey \\ University of California, Space Sciences Laboratory, Berkeley, CA 94720 \\ Received 1993 March 11; accepted 1993 May 19
}

\begin{abstract}
The recent observational data on gamma-ray bursts are reviewed. Burst time histories display features at the millisecond level which suggest a compact object origin. Lines in the energy spectra and spectral evolution point toward a Galactic neutron star origin, even though line features have not yet been confirmed in recent data. The Galactic distribution of burst sources, however, is both isotropic and sampled to its characteristic distance, making it unlikely that the sources are related to populations of Galactic neutrons stars we are familiar with. Counterpart searches, previously carried out years after the gamma-ray bursts, are now proceeding days after the events. Based on the current data, it is impossible to conclude whether bursters are Galactic, extragalactic, or both. Data being returned from current experiments, as well as data from new experiments to be launched in the next few years, may yet provide the answer.
\end{abstract}

Subject heading: gamma rays: bursts

\section{INTRODUCTION}

Galactic neutron stars were for a long time the accepted explanation for the origin of cosmic gamma-ray bursts. Following the confirmation by the Burst and Transient Source Experiment (BATSE) aboard the Compton Gamma-Ray Observatory of both an isotropic distribution of sources as well as a deficit of weak sources (Meegan et al. 1992), serious questions have arisen about this interpretation. It was generally expected that, as burst detectors became more sensitive, an anisotropy would be found along the Galactic disk, or possibly toward the Galactic center, consistent with the distributions of the Galactic neutron stars we are familiar with, such as radiopulsars, $\mathrm{X}$-ray binaries, or X-ray bursters. In addition, BATSE has not confirmed the observations of absorption and emission features in gamma-ray burst energy spectra (Teegarden et al. 1993), two of the strongest lines of evidence linking bursters to neutron stars.

There is, however, still evidence which indicates that at least some bursts are generated by Galactic neutron stars, and numerous models which attempt to reconcile theory and observations. This paper will review that evidence, and consider to what extent the BATSE results actually contradict a Galactic origin for bursters. In $\S 2$, the observations of rapid structure and periodicities in time histories are reviewed. Section 3 treats the energy spectra of bursts, particularly line features and the continuum evolution. Section 4 is devoted to the spatial distribution and the $V / V_{\max }$ statistic. Section 5 deals with the status of optical and X-ray counterparts.

This review is not exhaustive; further information on gamma-ray burst theories and observations have appeared in a recent conference proceedings (Paciesas \& Fishman 1992).

\section{TIME HISTORIES}

Gamma-ray burst durations may be as short as $8 \mathrm{~ms}$ or as long as $1000 \mathrm{~s}$, and display a corresponding diversity in their morphologies. The duration distribution displays possible evi- dence for two classes of events, having average durations around 0.1 and $10 \mathrm{~s}$ (Hurley 1992; Kouveliotou et al. 1993b; see Fig. 1); we will return to this in $\$ \S 3$ and 4 . Here we will discuss the fastest rise times, the shortest structures within a time history, the shortest durations, and the existence of periodicities.

The three fastest $e$-folding rise times appear to be (1) $\$ 7 \mathrm{~ms}$ for the 1993 January 31 burst (Fig. 2), (2) $0.5 \mathrm{~ms}$ for a soft gamma repeater observed on 1992 June 19 (Kouveliotou et al. 1993a), and (3) $\leq 0.2 \mathrm{~ms}$ for the 1979 March 5 event (Cline et al. 1979).

The two shortest structures within a time history are (1) a 0.2 ms spike observed by BATSE in the 1992 July 11 burst (Bhat et al. 1992), and (2) peaks lasting less than $5 \mathrm{~ms}$ in the 1984 December 15 event (Laros et al. 1985). The shortest burst durations are 1) $8 \mathrm{~ms}$ for the 1992 July 11 burst (Bhat et al. 1992), and 2) $\leq 16 \mathrm{~ms}$, for several events from SGR 180620 (Atteia et al. 1987a).

Fast structure in light curves need not imply a Galactic compact object origin. For example, a model of bursts which invoked superconducting cosmic strings was thought to be able to produce fast fluctuations (Babul, Paczyński, \& Spergel 1987; Paczyński 1988), and relativistic expansion or beaming might do the same. The simplest explanation, however, involves compact objects, although they need not be galactic; neutron star mergers appear to have sufficiently small dynamical timescales to produce fine time structure (Narayan, Paczyński, \& Piran 1992).

The only indisputable case of periodicity in a light curve remains the 1979 March 5 event, where an 8 s period pulse-interpulse structure was observed as a weak $(\approx 1 \%$ level $)$ afterglow following the main pulse (Barat et al. 1979). A hypothesis which was often advanced was that many, or even all burst light curves might display similar periodicities which would be undetectable for more typical (i.e., weaker) bursts, since instruments would not be sensitive enough to detect this weak a component. This now appears unlikely, however. Time his- 


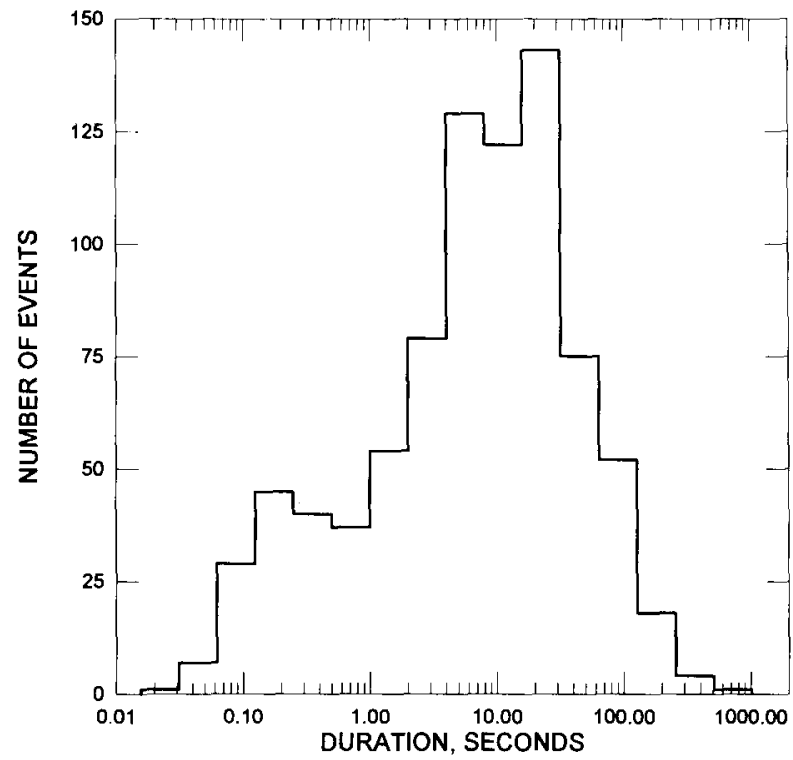

FiG. 1.-Duration distribution of 836 gamma-ray bursts observed by various instruments from 1978 to 1992 (Hurley 1992 and Kouveliotou et al., 1993b). The two peaks around 0.1 and $10 \mathrm{~s}$ may be taken as evidence for two classes of events.

tory studies using BATSE, which is sensitive enough to detect many weak, periodic afterglows at the $1 \%$ level, have revealed none (Kouveliotou et al. 1992).

\section{ENERGY SPECTRA}

The continuum spectra of gamma-ray bursts have now been measured from $\sim 1 \mathrm{keV}$ (e.g., Murakami et al. 1991) to well over $200 \mathrm{MeV}$ (Sommer et al. 1993). Like the time histories, the continua display a wide variety of morphologies, and variability has been observed at practically every time scale at which they have been measured. A simple representation of the photon continuum might be $E^{-1} \exp (-E / k T)$, with a $k T$ of several hundred $\mathrm{keV}$, up to several hundred $\mathrm{keV}$ or an $\mathrm{MeV}$, with a power law thereafter. The shorter bursts in Figure 1 tend to have harder spectra than the longer ones (Kouveliotou et al. 1993b). A clue to the origin of gamma-ray bursts may be found in observations of spectral evolution. Burst continua have been observed to evolve from (Murakami et al. 1991) and to (Yoshida et al. 1989) a shape consistent with $\mathrm{a} \approx 1.5$ $\mathrm{keV}$ blackbody. This spectral fit is not unique in either case, but if it is accepted, the ratio of the size to the distance of the emitting region may be deduced; it is consistent with $\approx 1 \mathrm{~km} / 1$ $\mathrm{kpc}$, strongly suggesting a Galactic neutron star as the source.

The strongest evidence for a compact object origin, however, has come from the observations of much-disputed line features in the energy spectra, namely absorption features around 20 and $40 \mathrm{keV}$, and emission features at energies around $400 \mathrm{keV}$. Both lines are often transient features in the changing continuum spectrum of a given burst. If the reality of these lines is accepted, the most likely interpretations are cyclotron scattering in teragauss magnetic fields, and gravitationally redshifted positron-electron annihilation, respectively. Much of the controversy surrounding the observations may be traced to the data analysis. A convincing case for spec- tral features may be made in one of several ways. For example, spectral forms with and without lines may be fitted, and an $F$-test performed to evaluate the significance of adding features to the continuum. As an alternative, a Bayesian inference method may be used (Laredo \& Lamb 1992). In either case, spectral deconvolution must be done to remove the instrumental signature. Table 1 summarizes the experiments which have reported line features over the years and whether a full statistical analysis has been published.

With the exception of the ICE experiment, which utilized a germanium detector, all the experiments in Table 1 were scintillators with only moderate energy resolution. This makes the question of statistical analysis even more crucial. As the table indicates, the Burst and Transient Source Experiment, which contains specially prepared spectroscopy scintillators, has not detected line features in any gamma-ray burst spectra to date. (Extensive searches have been carried out for the low-energy absorption lines, but considerably less effort has been expended to date on the search for high-energy emission lines). The sensitivity of this experiment to absorption features at energies $\gtrsim 20 \mathrm{keV}$ is approximately equal that of Ginga, which found evidence for lines in three out of 23 bursts. There are 52 bursts in the BATSE database with sufficient intensity to perform line searches. Adopting a $10^{-4}$ confidence level for line feature detection results in no detections; at the $10^{-3}$ level, one candidate appears. (The three Ginga detections were at $F$-test significance levels of $2.7 \times 10^{-3}, 2 \times 10^{-5}$, and $1.5 \times 10^{-7}$ ). Considering the statistics of the small numbers involved, there is no significant conflict yet between the old observations and the BATSE results.

\section{STATISTICAL PROPERTIES}

Unlike the spatial distributions of galactic neutron stars associated with radiopulsars, X-ray binaries, and X-ray bursters,

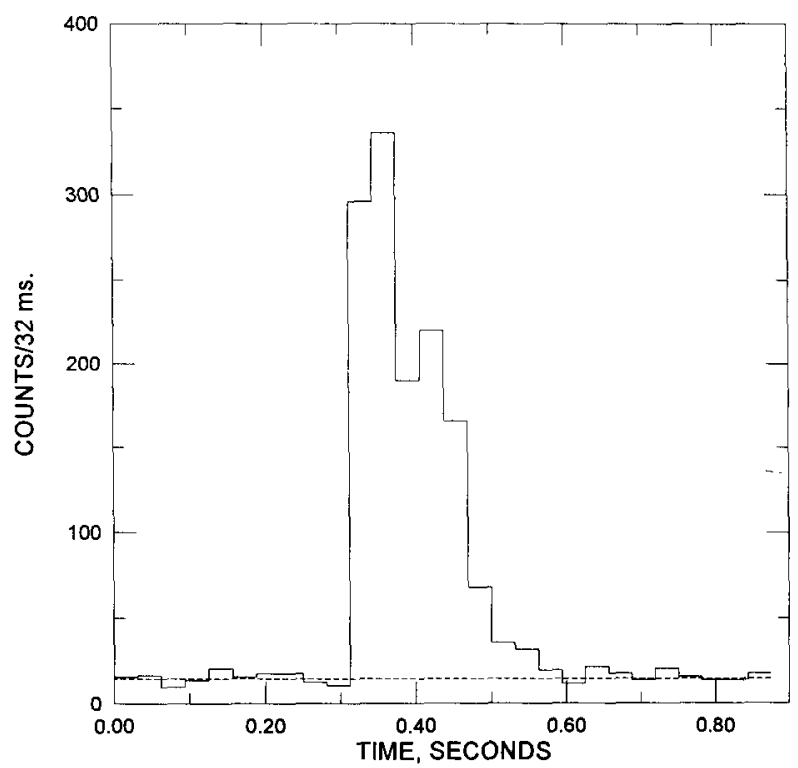

FIG. 2.-The first pulse of the 1993 January 31 burst as observed by Ulysses with $32 \mathrm{~ms}$ time resolution. An upper limit to the rise time of $\sim 7$ ms may be inferred from these data alone. 
TABLE 1

ABSORPTION AND EMISSION FEATURES IN GAMMA-RAY BURSTS

\begin{tabular}{|c|c|c|c|c|}
\hline Experiment & Reference & $\begin{array}{c}\text { Absorption } \\
\text { Features }\end{array}$ & $\begin{array}{c}\text { Emission } \\
\text { Features }\end{array}$ & $\begin{array}{c}\text { Statistical } \\
\text { Analysis }\end{array}$ \\
\hline Konus $11 / 12 \ldots$. & 1 & Yes & Yes & No \\
\hline ICE $3 \ldots \ldots \ldots$ & 2 & Not observable & Yes & Yes \\
\hline Konus $13 / 14$ & 3 & Yes & Yes & No \\
\hline$H E A O A-4$ & 4 & Yes & Yes & Yes \\
\hline Ginga ...... & 5 & Yes & Not observable & Yes \\
\hline Lilas (Phobos) ... & 6 & Yes & Not observable & No \\
\hline Apex (Phobos) ... & 7 & Not observable & Yes & No \\
\hline \multicolumn{5}{|l|}{ Konus-B } \\
\hline (Granat) & 8 & Yes & Yes & No \\
\hline BATSE ... & 9 & No & No & Yes \\
\hline
\end{tabular}

REFERENCES.-(1) Mazets et al. 1982; (2) Teegarden \& Cline 1980; (3) Mazets et al. 1983; (4) Hueter 1987; (5) Murakami 1991; (6) Barat et al. 1991; (7) Mitrofanov et al. 1991; (8) Golenetskii et al. 1991; (9) Teegarden et al. 1993.

the GRB spatial distribution is isotropic ( Fig. 3). As measured by BATSE, the dipole moment with respect to the Galactic center is $0.034 \pm 0.027$ and the quadrupole moment with respect to the plane is $0.316 \pm 0.014$ (vs. expected values of -0.014 and 0.329 , respectively, for isotropy when nonuniform sky coverage is taken into account). In addition, there is a pronounced deficiency of weak bursts, as measured by the $\langle\mathrm{V} /$ $V_{\max }>$ statistic: $0.324 \pm 0.014$ (Meegan et al. 1992).

If a single-component model (e.g., disk or halo only) for all bursters is assumed, and if only known matter distributions are considered, then Galactic compact objects are ruled out as sources (Mao \& Paczyński 1992). The Galactic disk is excluded because the weak source deficiency implies that we have sampled beyond the "edge" of the distribution, without detecting any quadrupole moment. The classical galactic halo cannot be the source because it would have to be spherically symmetric and have a minimum core radius of $\sim 18 \mathrm{kpc}$. If hypothetical matter distributions are allowed, however, an extended spherically symmetrical halo would satisfy the observa- tions; populating it probably requires that neutron stars be born there, rather than ejected from the disk, but there is some evidence that this may occur (Eichler \& Silk 1992), and some ideas about why these neutron stars might be more prolific producers of bursts than the Galactic disk component ( Li \& Dermer 1992). If two-component (disk + halo) models are considered, roughly one-third of the sources could be in the disk, at distances of around $300 \mathrm{pc}$, and the remainder in the halo, at $\sim 100 \mathrm{kpc}$, without conflicting with the observations (Lingenfelter \& Higdon 1992), and an even larger fraction could be coming from the disk if the halo population were contained in a spherical shell (Smith \& Lamb 1993). (Such a distribution might arise if bursters were neutron stars ejected from the Galactic disk at high velocity, which must age before passing through a temporary bursting stage). Suggestions of two burster distributions remain hypothetical, however; although the duration data $(\$ 2)$ display evidence for two components, they are not spatially distinct (Kouveliotou et al. 1993b).

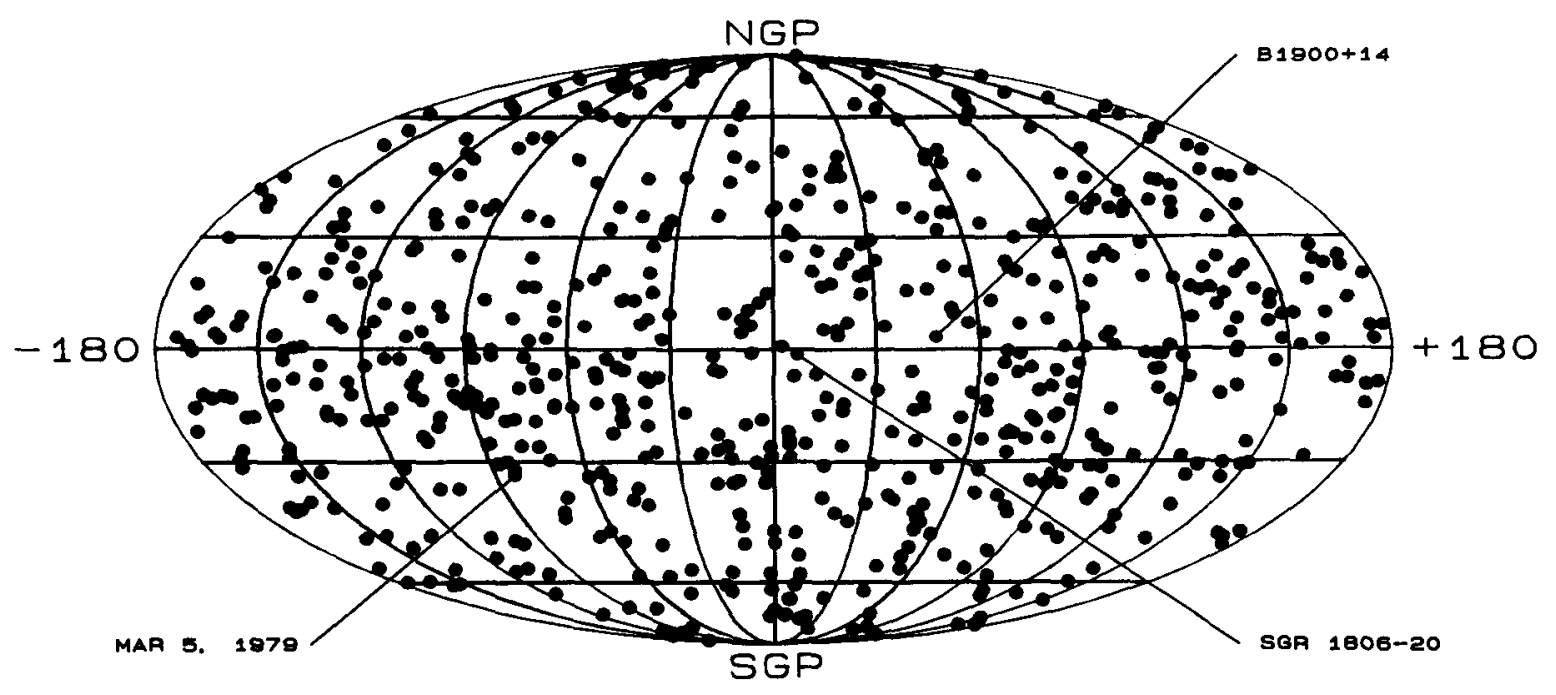

FIG. 3.-The distribution of cosmic gamma-ray bursts in Galactic coordinates. Approximately 450 are from the BATSE experiment (Fishman \& Meegan 1993), with the majority of the remainder from the interplanetary network (Atteia et al. 1987) and the KONUS experiment (Mazets et al. 1981). The positions of the only three repeating sources known (the soft gamma repeaters) are indicated. 


\section{COUNTERPART SEARCHES}

With the possible exception of the much debated March 5/N49 SNR association (Cline et al. 1982), no optical counterpart to a gamma-ray burster has been found. Interesting or suspicious properties of candidate objects might be jets, angular extent, a blue excess, variability, or possibly high proper motion. Galaxies are often found in gamma-ray burst error boxes, but the number of galaxies per square degree at high Galactic latitude exceeds the number of stars for magnitudes fainter than $\sim 22$. Since most searches go considerably deeper than this, at least several galaxies are expected in a 1 square arcminute error box. Indeed, in one cosmological model for bursters, the bursting objects have been ejected from their parent galaxies to a sufficient distance to make an association between the two difficult to determine (Narayan et al. 1992).

Several lines of evidence suggest that if bursters are neutron stars, they might be hot enough to produce quiescent EUV or $\mathrm{X}$-ray emission. One is that such objects are born hot, and cool rather slowly. But even if they have cooled, it is possible that the burst itself (Eichler \& Cheng 1989), or accretion preceding the burst (Pizzichini et al. 1986) could heat part or all of the object. Numerous X-ray searches have now been carried out with Einstein, EXOSAT, and ROSAT. A weak $\left(\approx 10^{-13}\right.$ ergs $\mathrm{cm}^{-2} \mathrm{~s}^{-1}$ ) Einstein source was detected in one case (Grindlay et al. 1982), although it was not confirmed by EXOSAT (Boer et al. 1988). Ten nondetections of various sources by Einstein and EXOSAT have also been reported (Pizzichini et al. 1986; Boer et al. 1988, 1991), at sensitivity levels between $10^{-13}$ and $10^{-12} \mathrm{erg} \mathrm{cm}^{-2} \mathrm{~s}^{-1}$. Observations with the Extreme Ultraviolet Explorer will take place within the next 6 months or so.

Practically all searches have taken place years after the bursts themselves, making it impossible to detect short-lived counterparts. A breakthrough was recently achieved with the third interplanetary network of gamma-burst detectors (Hurley et al. 1993). Optical, radio, and X-ray counterpart searches were carried out 12,13, and 19 days after a burst which occurred on 1992 May 1, and the ROSAT X-ray observation revealed a weak source $\left(\approx 10^{-13} \mathrm{erg} \mathrm{cm} \mathrm{cm}^{-2} \mathrm{~s}\right)$ in the error box (chance probability $\left.7 \times 10^{-4}\right)$. The possible relation between the X-ray source, the optical objects in the error box, and the burster is being examined. In particular, future $\mathrm{X}$-ray observations will determine the source spectrum to establish whether it is characteristic of a coronal or a nonthermal source, and the error box size will be reduced substantially to facilitate an optical identification. The time between the detection of a burst by the network and initiation of counterpart searches has since been reduced to several days, and multiwavelength observing campaigns can now be carried out more or less routinely.

\section{CONCLUSIONS}

Taken as a whole, the recent data on cosmic gamma-ray bursts neither directly support nor contradict the idea of a Galactic neutron star origin. They do indicate, however, that if bursters are Galactic, they represent a previously unknown spatial distribution. Despite the formidable energies required in extragalactic models, and the difficulties in extracting very high energy gamma-radiation from compact sources at large distances, such models provide a simple explanation for the statistical properties of gamma-ray burst sources and have become increasingly popular in recent years. Final confirmation or refutation of a Galactic origin may be a lengthy process. New observations of line features may take years, due to the small rate of observation of sufficiently intense events. Detection by BATSE is one possibility, but also, a high-resolution germanium spectrometer will soon be launched aboard the WIND spacecraft (Owens et al. 1991). Identification of a quiescent counterpart remains the elusive goal which could unambiguously determine the origin of gamma-ray bursts. Over the next few years, rapid searches of small interplanetary network error boxes will continue. Starting in 1994, the High Energy Transient Experiment spacecraft (Ricker et al. 1992) will provide not only the first multiwavelength observations of burst sources aboard a single spacecraft, but also near-instantaneous downlinking of precise burst positions, opening the way to far more rapid ground-based deep follow-up observations.

This work was supported in part by JPL contract 958056 , and NASA grant NAG5-1560.
Atteia, J.-L., et al. 1987a, ApJ, 320, L105

1987b, ApJS, 64, 305

Babul, A., Paczyński, B., \& Spergel, D. 1987, ApJ, 316, L49

Barat, C., et al. 1979, A\&A, 79, L24

1991, Planet. Space Sci., 39(1/2), 67

Bhat, P., et al. 1992, Nature, 359, 217

Boer, M., et al. 1988, A\&A, 202, 117

1991, A\&A, 249, 118

Cline, T., et al. 1979, ApJ, 237, L1

. 1982, ApJ, 255, L45

Eichler, D., \& Cheng, A. 1989, ApJ, 336, 360

Eichler, D., \& Silk, J. 1992, Science, 257, 937

Fishman, J., \& Meegan, C. 1993, private communication

Golenetskii, S., et al. 1991, Soviet Astron. Lett., 17(2), 83

Grindlay, J., et al. 1982, Nature, 300, 730

Hueter, G. 1987, Ph.D. thesis, University of California, San Diego,

Hurley, K. 1992, in AIP Conf. Proc. 265, Gamma-Ray Bursts, ed. W. Paciesas \& G. Fishman (New York: AIP), 13

Hurley, K., et al. 1993, in AIP Conf. Proc. 280, Compton Symp. (St.

Louis, 1992, Oct. 15-17) (New York: AIP), in press

\section{REFERENCES}

Kouveliotou, C. et al. 1992, in AIP Conf. Proc. 265, Gamma-Ray Bursts, ed. W. Paciesas \& G. Fishman (New York: AIP), 299 1993a, Nature, 362, 728

Laros, J., et al. 1985, Nature, 318,448

Li, H., \& Dermer, C. 1992, Nature, 359, 514

Lingenfelter, R., \& Higdon, J. 1992, Nature, 356, 132

Loredo, T., \& Lamb, D. 1992, in AIP Conf. Proc. 265, Gamma-Ray Bursts, ed. W. Paciesas \& G. Fishman (New York: AIP), 414

Mao, S., \& Paczyński, B. 1992, ApJ, 389, L13

Mazets, E., et al. 1981, Ap\&SS, 80, 3

1982, Ap\&SS, 82, 261

1983, in AIP Conf. Proc. 101, Positron-Electron Pairs in Astrophysics, eds. M. Burns, A. Harding, \& R. Ramaty (New York: AIP), 36 Meegan, C., et al. 1992, IAU Circ., No. 5478

Mitrofanov, I., et al. 1991, Planet. Space Sci., 39(1/2), 23

Murakami, T. 1991, Adv. Space Res., $11(8), 119$

Murakami, T., et al. 1991, Nature, 350, 592

Narayan, R., Paczyński, B., \& Piran, T. 1992, ApJ, 395, L83

Owens, A., et al. 1991, IEEE Trans. Nucl. Sci., 38(2), 559 
Paciesas, W., \& Fishman, G., eds. 1992, AIP Conference Proc. 265, Gamma Ray Bursts (New York: AIP)

Paczyński, B. 1988, ApJ, 335, 525

Pizzichini, G., et al. 1986, ApJ, 301, 641

Ricker, G., et al. 1992, in Gamma-Ray Bursts: Observations, Analyses, and Theories, ed. C. Ho, R. Epstein, \& E. Fenimore (Cambridge Univ. Press), 288
Smith, I., \& Lamb, D. 1993, ApJ, 410, L23

Sommer, M., et al. 1993, IAU Circ., No. 5707

Teegarden, B., \& Cline, T. 1980, ApJ, 236, L67

Teegarden, B., et al. 1993, in AIP Conf. Proc. Compton Symp. (St. Louis,

1992 October 15-17) (New York: AIP), in press

Yoshida, A., et al. 1989, PASJ, 41, 509 